\title{
Integrated wind farm layout and control optimization
}

\author{
Mads M. Pedersen and Gunner C. Larsen \\ Wind Energy Department, Technical University of Denmark, \\ Frederiksborgvej 399, 4000 Roskilde, Denmark \\ Correspondence: Mads M. Pedersen (mmpe@dtu.dk)
}

Received: 31 January 2020 - Discussion started: 24 February 2020

Revised: 3 July 2020 - Accepted: 1 October 2020 - Published: 12 November 2020

\begin{abstract}
The objective of this paper is to investigate the joint optimization of wind farm layout and wind farm control in terms of power production. A successful fulfilment of this goal requires the following: (1) an accurate and fast flow model, (2) selection of the minimum set of design parameters that rules or governs the problem, and (3) selection of an optimization algorithm with good scaling properties.

For control of the individual wind farm turbines with the aim of wind farm production optimization, the two most obvious strategies are wake steering based on active wind turbine yaw control and wind turbine derating. The present investigation is limited to wind turbine derating.

A high-speed linearized computational fluid dynamics (CFD) Reynolds-averaged Navier-Stokes (RANS) solver models the flow field and the crucial wind turbine wake interactions inside the wind farm. The actuator disc method is used to model the wind turbines, and utilizing an aerodynamic model, the design space of the optimization problem is reduced to only three variables per turbine - two geometric and one carefully selected variable specifying the individual wind turbine derating setting for each mean wind speed and direction.

The full design space is spanned by these $\left(2 N+N_{\mathrm{d}} N_{\mathrm{s}} N\right)$ parameters, where $N$ is the number of wind farm turbines, $N_{\mathrm{d}}$ is the number of direction bins, and $N_{\mathrm{S}}$ is the number of mean wind speed bins. This design space is decomposed into two subsets, which in turn define a nested set of optimization problems to achieve a significantly faster optimization procedure compared to a direct optimization based on the full design space. Following a simplistic sanity check of the platform functionality regarding wind farm layout and control optimization, the capability of the developed optimization platform is demonstrated on a Swedish offshore wind farm. For this particular wind farm, the analysis demonstrates that the expected annual energy production can be increased by $4 \%$ by integrating the wind farm control into the design of the wind farm layout, which is $1.2 \%$ higher than what is achieved by optimizing the layout only.
\end{abstract}

\section{Introduction}

The large-scale global deployment of wind energy is highly dependent on the cost of energy (COE), i.e. the profit of a wind power plant (WPP) over its lifetime as seen from an investor's perspective. Lowering the COE was previously addressed with the TOPFARM WPP layout optimization platform (Réthoré et al., 2013; Larsen and Réthoré, 2013). The platform is used to design a WPP with a minimal COE, for a given number of a predefined wind turbine (WT) type and an allowable area with a wind climate known a priori. Hence, it determines the optimal balance between WPP power produc- tion revenue on the one hand and, on the other hand, all relevant expenses. The considered expenses include WPP variable capital costs (i.e. capital costs that depend on the WPP layout), WPP operation and maintenance (O\&M) costs, and the cost of fatigue degradation of the individual components of all WTs in the WPP. The basic functionality of the TOPFARM platform was later extended by also including the number of WPP WTs as a design variable, and the performance of surrogate models, needed to facilitate the optimization algorithm used, was moreover improved by Mahulja et al. (2018). Because WT loading is included, the WPP WTs must be modelled as aeroelastic models (including individual 
WT control), and the inflow conditions to these are tightly coupled to the complex non-stationary wake-affected WPP flow field. Performing individual WT aeroelastic simulations for all the considered ambient wind speeds and wind directions in each layout configuration iteration is extremely costly in terms of computational efforts. Therefore, surrogate models are needed to link ambient WPP inflow conditions, WT location within the WPP, and WT response in terms of power production and (fatigue) loading.

However, WPP control aspects were not considered in the aforementioned WPP layout optimization platform. Fathy et al. (2001) present a purely theoretical analysis of coupled design and control of general physical systems. It was found that conventional sequential optimization processes are not guaranteed to find system-optimal designs. In this theoretical framework, a coupling term is introduced, which reflects the influence of plant dynamics and control on plant design. The necessary conditions for the combined plant design and controller optimality were investigated, and it was concluded that this term depends strictly on the gradients of the couplings with respect to the plant design variables, which is also intuitively clear. Therefore, for weak or no coupling, i.e. neglectable coupling constraint gradients, the plant design and controller optimization problems become separable and their sequential solution is equivalent to the combined optimum. In the case of a strong coupling, only design methods that include this interaction explicitly can produce system-optimal designs contrary to the sequential approach. A priori, however, it is not possible to evaluate whether the coupling between system design variables and system control variables is weak or strong for a complicated physical system like a WPP.

Fleming et al. (2016) and Gebraad et al. (2017) studied the optimization of layout and active wake control in terms of WT yaw-dictated wake deflection on a WPP with 60 WTs. In these studies, the wake effects are modelled with an augmented version of the N. O. Jensen model (Jensen, 1984) extended with an engineering model for wake deflection as caused by WT yaw misalignment. Fleming et al. (2016) consider an inflow wind speed of $8 \mathrm{~m} \mathrm{~s}^{-1}$ only and report a power gain of $2.3 \%$ for the optimized layout, $7.6 \%$ for the optimized yaw control, and $8.5 \%$ for the integrated layout and yaw control optimization result. Finally, Fleming et al. (2016) compare the integrated result, which requires 6900 CPU hours, with a sequential approach, which can be performed in "several hours by a single computer". They find that the integrated result is around $0.5 \%$ better than the results originating from the sequential approach. Gebraad et al. (2017) perform a three-step optimization: first the annual energy production (AEP) is increased by $1.5 \%$ by optimizing the layout considering one wind speed per wind direction only; then the WT positions and the yaw angle are optimized, again based on one wind speed per wind direction, which increases the AEP to $5.2 \%$ above the baseline. Finally, the WT yaw angles are optimized for all relevant wind speeds, raising the AEP to $5.3 \%$ above the baseline.

Another integrated approach is taken by Deshmukh and Allison (2017). They optimize a WPP system including WPP layout as well as WPP control facilitated by active wake control over the entire lifetime of the WPP. The optimal WPP system design is pursued using a quasi-steady empirical wake model (i.e. a deterministic wake, which expands downstream). The quasi-steady wake model is linearly superimposed on the undisturbed ambient flow fields including both mean wind shear and turbulence (presumably using only one turbulence seed and thereby one realization of the ambient stochastic turbulence field) to obtain a description of the WPP flow field. Surprisingly, a relationship between the atmospheric-boundary-layer (ABL) turbulence field and the introduced wake expansion factor is not established, although there is evidence that wake meandering, which depends on the site ambient turbulence field, is dictating the static downstream wake "envelope" (Machefaux et al., 2015). Deshmukh and Allison (2017) take a modelpredictive-control (MPC) approach that is specifically implemented using reduced-order state-space models of the individual WTs, which account for the tower fore-aft bending dynamics, the rotor rotational-speed dynamics, and the blade pitch dynamics. The active wake control includes both WT derating and wake deflection by WT yawing. The objective function is the WPP AEP, and two case studies indicate a significant improvement of the integrated system design compared to layout design only. No attempt was made to compare the full integrated system design approach with a sequential approach, in which first the layout was optimized and then, subsequently, the WPP control. Such a comparison would have contributed to quantification of the coupling terms, elaborated by Fathy et al. (2001), and, in the case of weak coupling, facilitated a reduction in the computational efforts needed to perform the system design optimization. The paper is ended with a comparison of the relative AEP effect of the derating and the yaw-wake-deflection strategy. It is concluded that wake deflection is of marginal importance compared to WT derating.

Based on a two-WT case, Andersen (2019) analysed active wake control using a high-fidelity computational fluid dynamics (CFD) large-eddy simulation (LES) solver, fully coupled with a modal-based aeroelastic tool including a full dynamic WT controller. Comparing a $35^{\circ}$ yaw case with the corresponding derating case, this study concludes that, for a given reduction in the upstream WT thrust, the yaw-wakedeflection strategy reduces the power production of the upstream WT more than the derating strategy. It is further concluded that the overall benefit of active wake deflection as well as WT derating is largely uncertain for a two-WT system.

Gebraad et al. (2015) also analysed a two-WT case by means of high-fidelity CFD simulations of the wind farm flow field coupled with simulations of the WT dynamics. 
They used the National Renewable Energy Laboratory's simulator for wind farm applications (SOWFA) to investigate two different derating strategies: (1) changing the collective blade pitch setting and (2) changing the tip speed ratio. They found that the power increase in the wake-affected WT was balanced by the decrease in the derated WT. This led to the conclusion that yaw wake deflection is more efficient than derating when quantified in terms of power production. Unfortunately, both of the investigated derating strategies are suboptimal as shown by Vitulli et al. (2019). The optimal derating strategy is the particular combination of collective pitch and tip speed setting, resulting in the lowest rotor thrust for a given WT power production. Using this optimal derating strategy, Vitulli et al. (2019) obtained a considerable power gain.

Regarding both numerical two-WT studies by Andersen (2019) and Gebraad et al. (2015), a full-scale study by van der Hoek et al. (2019), in which derating of the most upstream WT was investigated for a single row of five WTs, is particularly interesting. Their CFD simulations predicted a power increase of $5.6 \%$ for the row-aligned wind direction in the below-rated wind speed regime. This result was verified by a year-long field test campaign, where WT derating was turned on and off every half week. The derating was, for practical reasons, implemented as two pitch offsets (one for full-wake and one for partial-wake conditions). The results of the field test predicted an increase of $3.3 \%$ in AEP, i.e. close to the CFD simulations when taking the suboptimal pitch regulation as well as model and measurement uncertainties into account. Given that derating, based on pitch regulation only, is suboptimal, there is a potential for even larger gain by using the optimal combination of pitch and tip speed settings.

Large uncertainties, associated with both active-wakecontrol strategies (i.e. derating and yaw-based wake deflection) and, not least, among various simulation approaches as well as measurements, were also reported in Kheirabadi and Nagamune (2019). Important conclusions from this study are further that (1) full-scale tests provide the most conservative (i.e. less optimistic) evaluations of the potential of active wake control and (2) consistently "added layers of realism in terms of simulated wind conditions tend to deteriorate the performance of wind farm controllers".

Guided by (1) and (2), the present contribution to WPP system design optimization (i.e. WPP layout and control optimization) will seek to describe the complex inter-turbine aerodynamic interactions within a WPP as realistically as possible considering the computational resources needed for WPP optimization. This is done using an extremely fast fullblown CFD solver.
We will limit the scope to $\mathrm{AEP}^{1}$ system optimization; i.e. WT loading is excluded. We assume that WT characteristics for aggregated AEP estimates are sufficiently described in terms of their power and thrust coefficients, which implicitly include the relevant structural dynamics of a particular WT as e.g. crucial blade bending and torsion dynamics for big modern WTs with flexible blades. Encouraged by the results obtained by Deshmukh and Allison (2017), Andersen (2019), and van der Hoek et al. (2019), we will limit WPP active wake control to WT derating and leave inclusion of yaw-dictated wake deflection for a future study.

The research challenges dealt with in the present paper can be summarized as follows:

1. Investigate WPP system optimization based on fullblown CFD simulations of the complex WPP flow field with its complicated WT wake interactions.

2. Analyse and indicate the importance of the system coupling terms mentioned in Fathy (2001) - or more specifically their gradients with respect to the WT positions.

3. Evaluate the AEP improvement potential accompanying the integrated system approach with a focus on individual WT derating based on analysis of an existing offshore WPP.

Section 2 describes the simulation platform including all relevant models, while Sect. 3 presents a simple and illustrative application example as a sanity check. The Lillgrund case study is described in Sect. 4. First the layout-control coupling is analysed by a one-row WPP example. Based on the results of this study, a system optimization of the Lillgrund WPP is subsequently performed. The paper is concluded in Sect. 5, where future work is also identified.

\section{The platform}

Overall, the integrated layout and WPP control optimization platform is based on a fusion of TopFarm2 (Pedersen et al., 2019a); the DTU wake framework, PyWake (Pedersen et al., 2019b); and a dedicated aerodynamic rotor model. TopFarm2, which is the DTU open-source WPP optimization framework, utilizes the open-source framework for multidisciplinary design, analysis, and optimization, OpenMDAO (Gray et al., 2019), to find the optimal set of design variables, i.e. WT positions and control settings in a sequential or nested workflow. PyWake is the DTU open-source AEP calculator including a collection of stationary wake models. PyWake is used to establish the AEP objective function

\footnotetext{
${ }^{1}$ Restricting the objective function to power production is a major simplification compared to the approach taken in Réthoré et al. (2013), Larsen and Réthoré (2013), and Mahulja et al. (2018) because (1) aeroelastic modelling of the WPP WTs is circumvented, (2) a stationary description of the wake-affected WPP flow field suffices, and (3) no cost models are needed.
} 
needed in TopFarm2, which in this study is based on the linearized CFD Reynolds-averaged Navier-Stokes (RANS) wake model, Fuga (Ott et al., 2011).

A simplified version of the present platform, excluding WPP layout optimization and thus only including WPP control optimization, is described by Vitulli et al. (2019). In its most general formulation, this open-loop WPP control optimization platform deals with two design parameters per WT - the tip speed ratio, $\lambda$, and the collective pitch angle, $\alpha$, both conditioned on the wind direction and wind speed. However, using a case study Vitulli et al. (2019) justifies that the design space, without loss of generality, can consistently be collapsed to only one parameter for each WT. This parameter reflects the desired derating and maps to a unique combination of collective pitch and tip speed ratio, $\left(\alpha_{\mathrm{C}_{\mathrm{t}}}, \lambda_{\mathrm{C}_{\mathrm{t}}}\right)$, which results in the smallest possible thrust coefficient, $C_{\mathrm{t}}$, conditioned on the requested power coefficient, $C_{\mathrm{p}}$. For the sake of efficiency, we will take advantage of this finding in designing the present platform, thus resulting in three design parameters for each WT - two layout coordinates and the unique set $\left(\alpha_{\mathrm{C}_{\mathrm{t}}}, \lambda_{\mathrm{C}_{\mathrm{t}}}\right)$ resulting from the unique functional relationship $\alpha_{\mathrm{C}_{\mathrm{t}}}\left(\lambda_{\mathrm{C}_{\mathrm{t}}}\right)$.

In summary, the present integrated system optimization platform consists of four main components:

1. a CFD solver modelling the steady-flow field within a WPP; the ambient mean wind shear and turbulence characteristics are specified in terms of a terrain roughness height conditioned on wind direction, which implicitly dictates the ambient turbulence conditions via the turbulence closure of the CFD model;

2. an aerodynamic part, which models the WT power and thrust characteristics based on a detailed aeroelastic model of the WT; this model incorporates a description of both structural and aerodynamic properties of the WT with predefined settings for rotational speed and the collective pitch angle conditioned on the rotor inflow conditions; however, only steady WT deflections are accounted for in defining the rotor aerodynamic characteristics for the present purpose; this model is in turn used to establish an accurate and fast surrogate model to facilitate an efficient optimization process;

3. a WPP AEP performance metric defining the optimization objective, including possible constraints, as based on information available a priori on the mean wind direction probability density function (pdf) and the mean wind speed pdf conditioned on the wind direction of the site; and

4. an optimization platform that computes the optimal system performance in terms of the WPP AEP metric while satisfying the site area and minimum wind turbine separation constraints.
In the following each of these four key elements is described in some detail.

\subsection{The CFD solver}

Typically, an optimization of the control settings for a WPP requires 200-1000 power evaluations for each mean wind speed and direction. To calculate a proper AEP metric, we use 23 speeds and 360 directions; i.e. 1.6-8.2 million flow field computations are needed to optimize the control settings for a given WPP layout. Obviously, this puts excessively high demands on the computational speed of the flow solver.

The linear CFD RANS solver, Fuga (Ott et al., 2011), is extremely fast, has previously compared well with fullscale measurements (Peña et al., 2018; van der Laan et al., 2019), and is thus considered ideal for this task. The governing Navier-Stokes equations, neglecting the Coriolis forcing, are consistently linearized using a formal perturbation expansion and subsequently retaining only the first-order perturbation terms. Thus, mass conservation is identically satisfied; momentum conservation is satisfied to first order; and the resulting WPP fields are divergence free, as they should be for an assumed incompressible flow. The resulting equations are in turn conveniently formulated and solved in a mixedspectral domain for efficiency reasons. The velocity perturbation around a single WT in the physical domain is derived from Fourier components of the mixed-spectral solution using a fast inverse Fourier integral transform and stored in a system consisting of both general and WT-specific look-up tables, which facilitates the extreme computational speed of the solver. Because of the linearity of the model, wakes from multiple upstream WTs can consistently be superimposed to construct the flow field further downstream. From an efficiency perspective, this is a big advantage.

The WTs are modelled as actuator discs, which in general can be vertically inhomogeneous but are often assumed to be uniform in wake studies. The actuator discs embedded in the flow field represent the rotor drag forces, which in turn are responsible for the creation of rotor downstream wakes. The specifications of the individual actuator discs are based on detailed aerodynamic models of the WPP rotors as accounted for in Sect. 2.2. The WPP wind field, impinging on an arbitrary WT in the WPP, depends on the ambient wind field and wakes from relevant upstream WTs linearly superimposed.

The inflow conditions, i.e. mean wind speed and direction, are assumed to be horizontally homogeneous over the spatial extend of the WPP. More specifically, neutral atmospheric boundary conditions are assumed, meaning that a logarithmic mean wind shear profile applies. The characteristics of the shear profile is thus in turn defined by a terrain roughness length and the friction velocity $u_{*}$. For neutral atmospheric conditions, Monin-Obukhov scaling dictates the standard deviation of the velocity fluctuations to be invariant through the atmospheric boundary layer and proportional to the friction velocity. The turbulence inflow is thus ex- 
pressed in terms of the same input parameters as the mean wind shear field. For the Lillgrund site, a roughness length of $z_{0}=0.03 \mathrm{~m}$ is used, which results in an inflow turbulence intensity of approximately $12 \%$. This is at the high end for an offshore location and relates to the proximity of the Lillgrund site to urban areas. No attempt was made to link the roughness length to inflow wind speed, because site measurements have shown only marginal variations in the turbulence intensity with wind speeds below the rated wind speed.

For each wind direction, the local wind speed, i.e. ambient wind speed minus the sum of deficits from upstream turbines, the power production, and the thrust coefficient as well as the wake deficits at downstream WT positions are evaluated starting with the most upstream WT position and continuing in the downstream order.

\subsection{The aerodynamic WT model}

As mentioned, we consider detailed aerodynamic rotor performance expressed in terms of power- and thrust coefficients as fully satisfactory for WT AEP simulations.

Initially, the power and thrust coefficients of the rotor are modelled using HAWCStab2 - a linearized aero-servoelastic code designed for stability analysis and steady-state simulation of WTs (Hansen et al., 2017). HAWCStab2 relies on an extended formulation of the traditional blade element momentum (BEM) approach (Madsen et al., 2007), and consequently detailed geometric and aerodynamic input is required, e.g. the blade planform and twist distribution as well as blade aerodynamic properties in terms of aerodynamic coefficients over the blade length. In the present application, HAWCStab2 uses a fully flexible WT model formulation to account for the equilibrium-static wind-speed-dependent deflections of the WT main components and thus the potential effects on the WT thrust and power performance.

For traditional layout optimization without WPP control, the WPP production is implicitly based on WTs running at maximum $C_{\mathrm{p}}$. For the present application, which aims at system-optimal design, the aerodynamic modelling includes a WT derating feature, which links to a unique set of the tip speed ratio and collective pitch angle, $\left(\alpha_{\mathrm{C}_{\mathrm{t}}}, \lambda_{\mathrm{C}_{\mathrm{t}}}\right)$. Consequently, the aerodynamic WT model must facilitate computation of $C_{\mathrm{t}}$ and $C_{\mathrm{p}}$ conditioned on these design variables. Assuming zero yaw error, the tip speed ratio, $\lambda$, is defined as

$\lambda \equiv \frac{R \Omega}{U}$,

where $R$ is the rotor radius, $\Omega$ denotes the rotor speed, and $U$ is the hub-height mean wind speed.

The conditional dimensionless rotor thrust and power coefficients are defined as

$C_{\mathrm{t}}(U \mid \alpha, \lambda) \equiv \frac{T_{\mathrm{WT}}(U \mid \alpha, \lambda)}{\frac{1}{2} \rho A U^{2}}$ and

$C_{\mathrm{p}}(U \mid \alpha, \lambda) \equiv \frac{P_{\mathrm{WT}}(U \mid \alpha, \lambda)}{\frac{1}{2} \rho A U^{3}}$,

respectively, where $T_{\mathrm{WT}}$ is the rotor thrust force; $P_{\mathrm{WT}}$ is WT power production; $\rho$ is the air density; and $A$ is the rotor area, which depends on both the rotor tilt $\left(\theta_{\mathrm{t}}\right)$ and the blade-coning $\left(\theta_{\mathrm{c}}\right)$ angles as

$A=\pi\left(R \cos \theta_{\mathrm{c}} \cos \theta_{\mathrm{t}}\right)^{2}$.

In this context, $P_{\mathrm{WT}}$ and $T_{\mathrm{WT}}$ are obtained from HAWCStab2 simulations of the Siemens SWT-2.3-93 WT, which operates at the Lillgrund WPP; see Sect. 4. The steady-state power and thrust have been simulated for a range of collective pitch and rotor speed settings in a uniform flow field of $8 \mathrm{~m} \mathrm{~s}^{-1}$. In principle such steady-state parameter sweep simulations must be performed for all relevant mean wind speeds to account for the steady-state blade deflections. However, assuming that these deflections have only a minor effect on the steady-state power and thrust performance of the WT in question, then one mean wind speed suffices. This is justified under the assumption that the thrust scales with $U^{2}$ and the thrust coefficient is normalized with $U^{2}$, whereas the power scales with $U^{3}$ and the power coefficient is normalized with $U^{3}$.

Note from Eq. (1) that for a fixed wind speed, a variation in rotor speed corresponds to a variation in $\lambda$. Thus, from the above-described simulation outputs, the power and thrust coefficients are easily calculated as a function of the tip speed ratio and the collective pitch via Eqs. (1)-(4); see Fig. 1.

The results shown in Fig. 1 can be used for the entire range of mean wind speeds requested for the system optimization; see Eq. (1). This is convenient from a computational point of view and thus consolidates the status of the tip speed ratio as a design variable as an appropriate choice.

Another important computational simplification is, as previously mentioned, the reduction from two control design variables per WT to one control design variable per WT. This reduction is based on the previously mentioned findings by Vitulli et al. (2019) showing that optimal derating is obtained by selecting the unique set of design variables $\left(\alpha_{\mathrm{C}_{\mathrm{t}}}, \lambda_{\mathrm{C}_{\mathrm{t}}}\right)$, which, for a given derating (i.e. power production reduction), corresponds to the smallest possible thrust. This condition, which is also intuitively clear, provides a unique relationship between $\alpha_{\mathrm{C}_{\mathrm{t}}}$ and $\lambda_{\mathrm{C}_{\mathrm{t}}}$ and justifies the reduction in design space to one control variable per WT, conditioned on ambient mean wind direction and mean wind speed.

As a consequence of the control design space collapse, a specific derating factor corresponds to a deterministic path through the original $(\alpha, \lambda)$ design space, where the points on this path correspond to certain mean wind speeds. Note that these paths are constrained by the minimum and maximum rotor speed limits as well as the maximum power limit; see Fig. 2. 

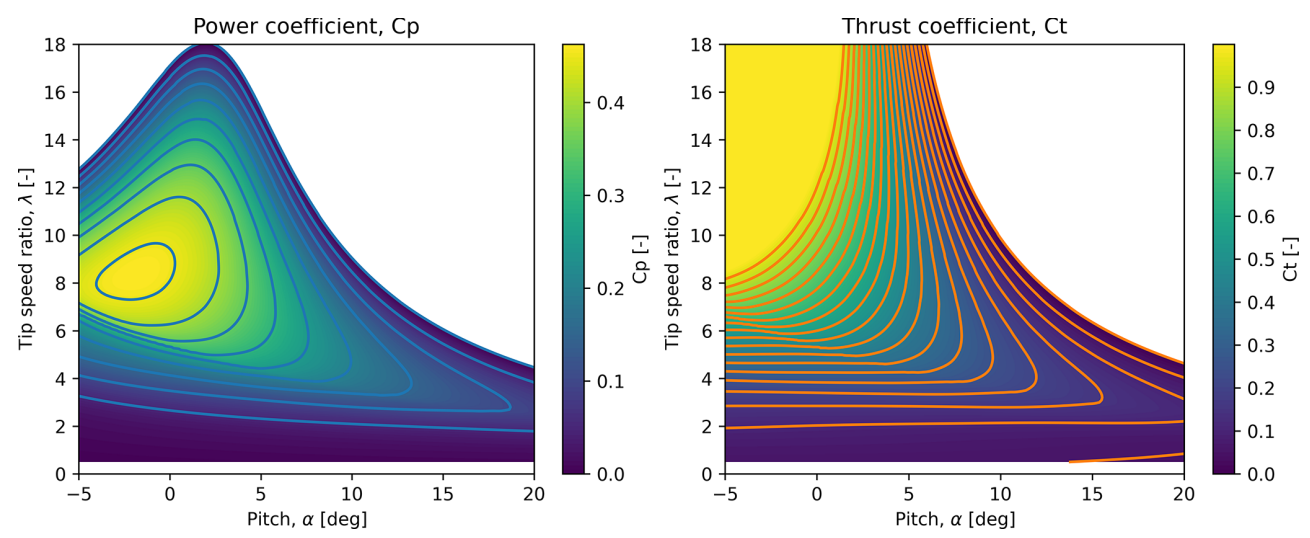

Figure 1. Power and thrust coefficients as a function of tip speed ratio and collective pitch angle, based on HAWCStab2 simulations of a Siemens SWT-2.3-93 WT.
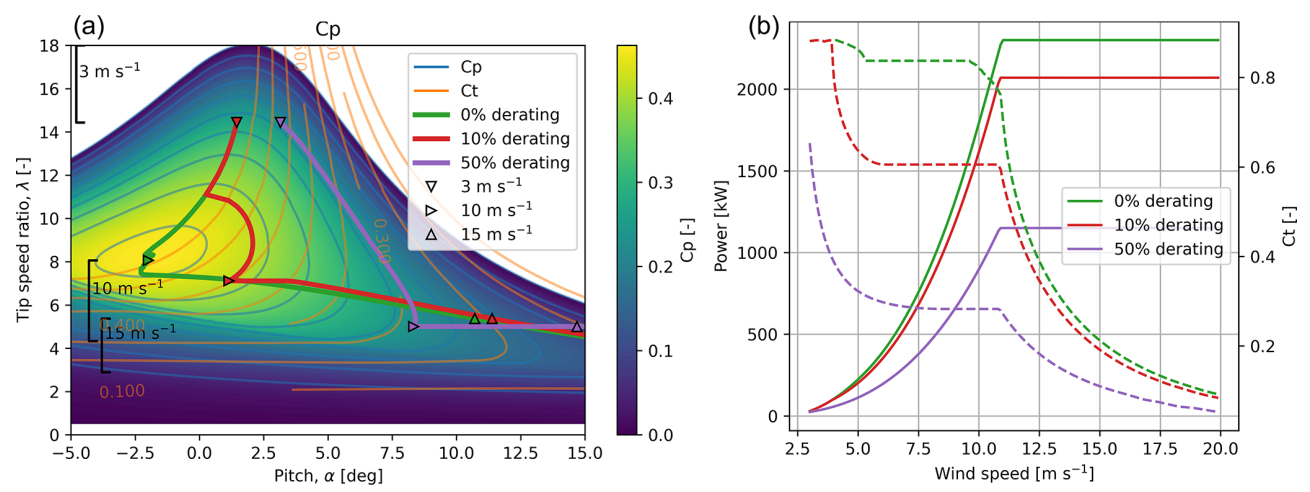

Figure 2. (a) $C_{\mathrm{p}}$, (background colour and blue contours) and $C_{\mathrm{t}}$ (orange contours) plotted as a function of tip speed ratio, $\lambda$, and collective pitch setting, $\alpha$. The green, red, and purple lines expose the $\left(\alpha_{\mathrm{C}_{\mathrm{t}}} \lambda_{\mathrm{C}_{\mathrm{t}}}\right)$ relation for $0 \%, 10 \%$, and $50 \%$ derating, respectively. These relations are plotted for a range of wind speeds $\left(3,10\right.$, and $15 \mathrm{~m} \mathrm{~s}^{-1}$ are marked) satisfying the rotor speed limits (indicated on the left-hand side of the panel for 3,10 , and $15 \mathrm{~m} \mathrm{~s}^{-1}$ ) as well as the maximum power limit. (b) The corresponding power (solid) and $C_{\mathrm{t}}$ (dashed) curves plotted as a function of wind speed. These figures are based on HAWCStab2 simulations of a Siemens SWT-2.3-93 WT model.

The last step needed to prepare for an efficient optimization procedure is to transform the above-described aerodynamic rotor computations into a surrogate model, which maps mean hub wind speed and the requested derating factor into a power production coefficient and a thrust coefficient conditioned on the operational settings, i.e. $C_{\mathrm{p}}(U \mid \alpha, \lambda)$ and $C_{\mathrm{t}}(U \mid \alpha, \lambda)$. The surrogate thereby establishes the link between the derating settings, to be specified by the control optimizer, and the characteristics of the uniformly loaded actuator discs needed by the flow solver. Note that controllerspecific constraints such as the tower exclusion zone and smooth transition between regions as well as controller implementation issues are not taken into account. At present, it has not been found essential to model the actuator discs as vertically inhomogeneous, although this is possible within the framework.

\subsection{The AEP performance metric and constraints}

The objective function defined for the present optimization platform is the AEP of the WPP. Financial costs of the internal WPP power grid, access roads, foundation, etc. are not considered, which in turn means that the positions of the individual WPP WTs are only constrained by the minimum allowable distance to the nearest neighbouring WT and the line of demarcation defining the permissible WPP area. Considering two rotor diameters (2D) to be the minimum realistic WT interspacing distance, this minimum spacing constraint has been selected for all show cases presented in this paper. The permissible WPP area for the Lillgrund case is the stylized convex shape of the Lillgrund reef. Finally, we have incorporated two additional constraints associated with the operational conditions of the Lillgrund Siemens SWT-2.3-93 WT used in all cases: $\Omega \epsilon[9 \mathrm{rpm}, 16 \mathrm{rpm}]$ and $\alpha \epsilon\left[-2^{\circ}, 90^{\circ}\right]$.

In each iteration of the optimization procedure, the objective function - in this case the AEP performance metric must be computed. Computational efficiency is in particular 
needed for the present CFD-based approach, and maximum efficiency is assured through implementation of the "shortcuts" described in Sect. 2.2.

For a given layout (i.e. associated with a given iterative step in the layout optimization process), the WPP AEP, $P_{\mathrm{AEP}}$, is estimated from

$P_{\mathrm{AEP}}=T \sum_{i=1}^{N} \int_{0^{\circ}}^{360^{\circ}} \int_{U_{\text {in }}}^{U_{\text {out }}} P_{i}(U \mid \theta) f_{U}(U \mid \theta) f_{\theta}(\theta) \mathrm{d} U \mathrm{~d} \theta$

in which $U$ denotes the undisturbed ambient hub-height mean wind speed and $P_{i}(U \mid \theta)$ is the production (in watts) of the $i$ th WT at ambient hub-height mean wind speed, $U$, and associated operating conditions dictated by the internal WPP flow field. $f_{U}(U \mid \theta)$ is the ambient hub-height mean wind speed pdf, conditioned on the ambient mean wind direction (i.e. often a two-parameter Weibull distribution), and $f_{\theta}(\theta)$ is the ambient mean wind direction pdf. Assuming SI units, $T$ is the number of seconds corresponding to 1 year and $N$ is the predefined number of WTs within the WPP considered.

In practice, Eq. (5) is discretized to facilitate evaluation of the involved integrals. In the succeeding case studies, a directional discretization of $1^{\circ}$ was used combined with an ambient mean wind speed discretization of $1 \mathrm{~m} \mathrm{~s}^{-1}$.

\subsection{Optimization setup}

Overall, there are three common ways to design the WPP system optimization. The most elaborate of these is to design the fully integrated approach by involving all design variables simultaneously - the one-step approach. The layoutoptimization-related design variables amount to two (i.e. the WT position in a Cartesian coordinate system) per WT. The WPP control optimization, conditioned on ambient mean wind direction and mean wind speed, requires, utilizing the design space collapse described in Sect. 2.2, one design variable per WT. However, because the AEP computation requires all wind directions and all wind speeds to be accounted for, the control-related design variables amount to $N_{\mathrm{d}} N_{\mathrm{s}}$ per WT. Here $N_{\mathrm{d}}$ is the number of ambient inflow directions and $N_{\mathrm{S}}$ is the number of ambient mean wind speeds considered in the discrete version of Eq. (5). Thus, in total the number of design variables amounts to $N\left(2+N_{\mathrm{d}} N_{\mathrm{s}}\right)$. This is clearly infeasible within the present framework - even when utilizing a high-performance computing cluster.

An alternative and more efficient strategy for a fully integrated system optimization is a two-step nested approach, in which, for each optimization step, first the layout is advanced and then, based on this iteration of the layout, the associated optimal control schedule, conditioned on ambient mean wind speed and direction, is determined. Merging the sequentially determined WPP layout and associated optimal control schedule, the AEP estimate, associated with the actual iterative step, can be evaluated. The associated workflow is illustrated in Fig. 3.

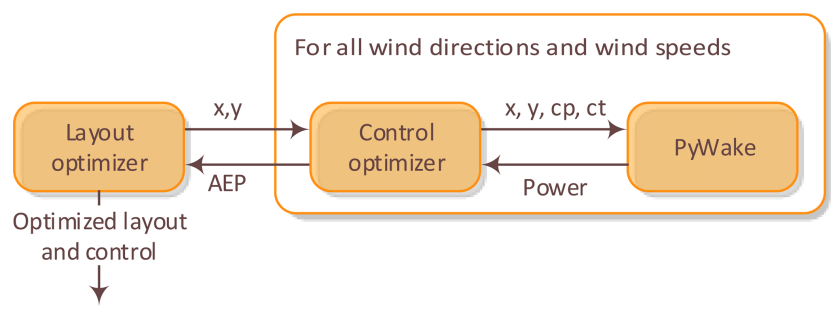

Figure 3. Nested optimization workflow. The control settings are optimized in every layout iteration.

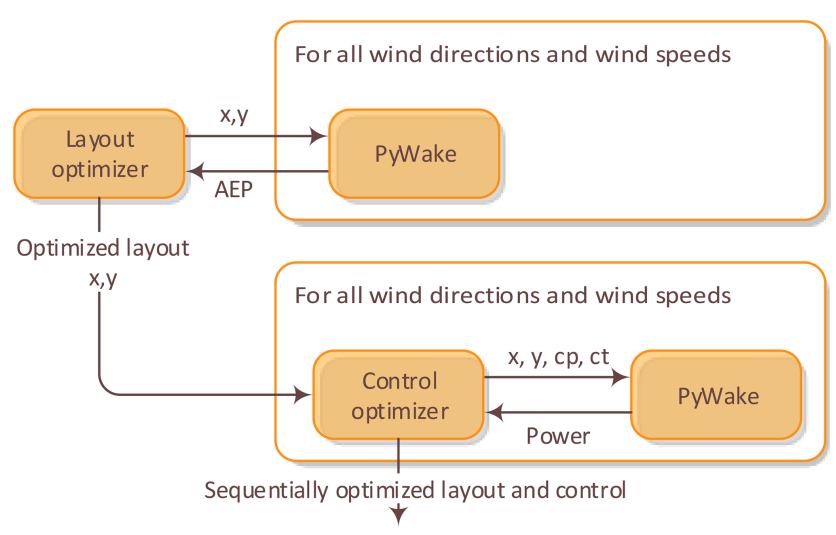

Figure 4. Sequential optimization workflow. The control settings are optimized one time only, after the optimal layout is found.

Both the one-step optimization strategy and the two-step nested optimization strategy are fully integrated strategies, which eventually will lead to the same result.

If the optimal system design is separable, in the sense that only a weak coupling exists between the layout and the WPP control optimization, the problem can be significantly simplified. This will be quantified in Sects. 3.3 and 4.1 using two demonstration cases. The significant reduction in computational complexity is obtained taking a two-step sequential approach by approximating a weak system coupling with no system coupling. The sequential workflow, in which the conventional "greedy" individual WT control settings are used for the WPP layout optimization, is succeeded by an optimization of the WPP control scheduling conditioned on both ambient mean wind speed and direction. Thereby, the greedy WT control settings are replaced by optimized "collaborative" WT settings to the benefit of the WPP AEP. The workflow associated with this sequential strategy is shown in Fig. 4.

The merger of these two optimization steps makes up the optimized system design and is in essence a sequential application of the TopFarm2 (Pedersen et al., 2019a) layout platform and the open-loop WPP control scheduling platform described by Vitulli et al. (2019).

The layout is optimized using a combination of randomsearch and gradient-based (SLSQP - sequential least-squares programming) optimization. The random-search algorithm, 


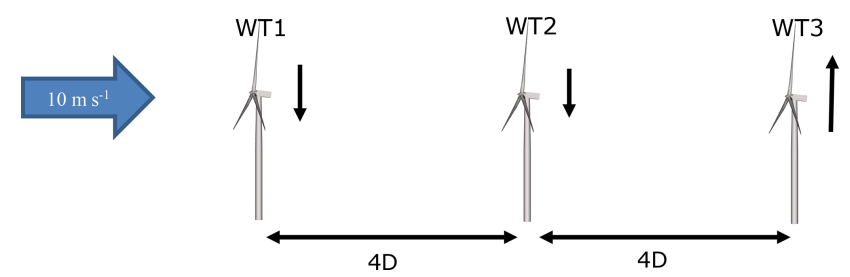

Figure 5. Three-WT row used for sanity check of the control optimization. Reduced WT production, caused by derating, is indicated by an arrow pointing downwards; increased WT production, caused by optimized WPP control, is indicated by an arrow pointing upwards.

described by Feng and Shen (2015), does not get stuck at local optima and is consequently suitable to finding a good global solution, while the gradient-based optimizer, applied subsequently, is used to trim the random-search solution to the nearest optima. In this setup, the gradients are approximated by a finite-difference approach. For a complex non-convex optimization problem, a global optimum cannot theoretically be ensured, but running numerous random sequences converging to almost identical results gives confidence in the result being close to the global optimum.

The WPP control scheduling optimization problem has in general only a few local optima and can therefore easily be solved by the gradient-based optimizer using gradients computed via finite difference. This control optimization is, however, rather time-consuming, as the WT control settings must be optimized for all $360 \times 23$ combinations of wind directions and wind speeds; see Table 1 . These combinations are, fortunately, independent, and the workflow is therefore suitable for parallel computation. For the current study, a parallel workflow utilizing $360 \mathrm{CPUs}$ (i.e. corresponding to a $1^{\circ}$ mean wind direction resolution) has been set up, where each CPU optimizes all WT control settings for one wind direction. Table 1 gives an idea of the computational resources needed for the case studies described in Sects. 3 and 4.

\section{Sanity check}

To check the overall behaviour of the optimizers, a sanity check on a simple illustrative example, consisting of a row with three Siemens SWT-2.3-93 WTs, has been performed. This case is selected, because it can be solved via "brute force" and because the results are easily visualized.

\subsection{Control optimization}

The sanity check of the control optimization is performed on a simple example consisting of three WTs in a row, separated by $4 \mathrm{D}$ and with a uniform inflow of $10 \mathrm{~m} \mathrm{~s}^{-1}$ aligned with the row; see Fig. 5.

Figure 6 shows the power produced by the three WTs as a function of the derating of the two upstream WTs. In panel a, it is seen that the power for the most upstream WT, WT1, only depends on its own derating setting. The power of WT2, on the other hand, depends on the derating of both itself and of WT1 (panel b). Finally, it is seen that WT3, obviously, produces the most if both WT1 and WT2 are derated $100 \%$ (panel c).

The total power produced by the three WTs is seen in Fig. 7, and it appears that the total power can be increased by $4.01 \%$ if WT1 is derated by $7 \%$ and WT 2 is derated by $5 \%$.

\subsection{Layout optimization}

A sanity check of the layout optimizer is also performed on the three-WT row. In this case, the position of WT2 is allowed to vary between 2 and $6 \mathrm{D}$ behind WT1. Figure 8 shows the individual relative power production of the three WTs as well as the total power production as a function of the position of WT2 in a uniform flow of $10 \mathrm{~m} \mathrm{~s}^{-1}$ aligned with the row. As expected, WT1 is unaffected by the position of WT2, while the power production of WT2 increases with the distance to WT1 and vice versa for the power of WT3. Finally, the total power production is seen to increase slightly when WT2 is moved downstream.

For other wind speeds, however, the picture is quite different, as seen in Fig. 9. The optimal position thereby depends on the wind speed distribution, which links to the dependence of $C_{\mathrm{p}}$ and $C_{\mathrm{t}}$ on wind speed with the hub-height mean wind speed. Plotting the relative AEP computed using the Weibull distribution associated with westerly winds at the Lillgrund wind farm (c.f. the wind rose shown in Fig. 12) reveals that the optimal spacing, under these conditions, is very close to 4D.

\subsection{Combined layout and control optimization}

The performance of the integrated layout and control optimization is illustrated in Fig. 10. The blue line indicates the relative AEP of the three WTs as a function of the position of WT2 in the case that all WTs are operated with greedy settings (i.e. no derating). This is the base case. The optimal position of WT2 is found to be 3.96 D downstream of WT1. Applying layout-dependent optimal derating of WT1 and WT2 (orange curve) sequentially increases the AEP of the initial layout by $2.221 \%$. Finally, the AEP is seen to increase only infinitesimally (i.e. increasing from $+2.221 \%$ to $+2.226 \%$ ) when applying integrated two-step nested system optimization. For the investigated simplistic case, this result indicates a very weak system coupling between WPP layout and control optimization.

\section{The Lillgrund case study}

The Lillgrund WPP is located in Øresund between Denmark and Sweden and consists of 48 Siemens SWT-2.3-93 WTs, 
Table 1. Overview of time consumption of the AEP calculation, the control optimization, and the layout optimization. WD is wind direction; WS is wind speed.

\begin{tabular}{lll}
\hline & $\begin{array}{l}\text { Row of 8 WTs } \\
\text { (1 D layout, 1 WD, 23 WSs) }\end{array}$ & $\begin{array}{l}\text { Lillgrund, 48 WTs, } \\
\text { (2 D layout, 360 WDs, 23 WSs) }\end{array}$ \\
\hline PyWake, AEP calculation & $0.002 \mathrm{~s}$ & $0.52 \mathrm{~s}$ \\
\hline Control optimization & $3.5 \mathrm{~s}$ & $\begin{array}{l}15 \mathrm{~h}(1 \mathrm{CPU}) \\
4 \text { min (360 CPUs) }\end{array}$ \\
\hline Layout optimization & $3.4 \mathrm{~s}$ & $\begin{array}{l}2.8 \mathrm{~h} \text { (random search; 1 CPU) }+ \\
1.2 \mathrm{~h} \text { (gradient based; } 1 \mathrm{CPU})\end{array}$ \\
\hline
\end{tabular}
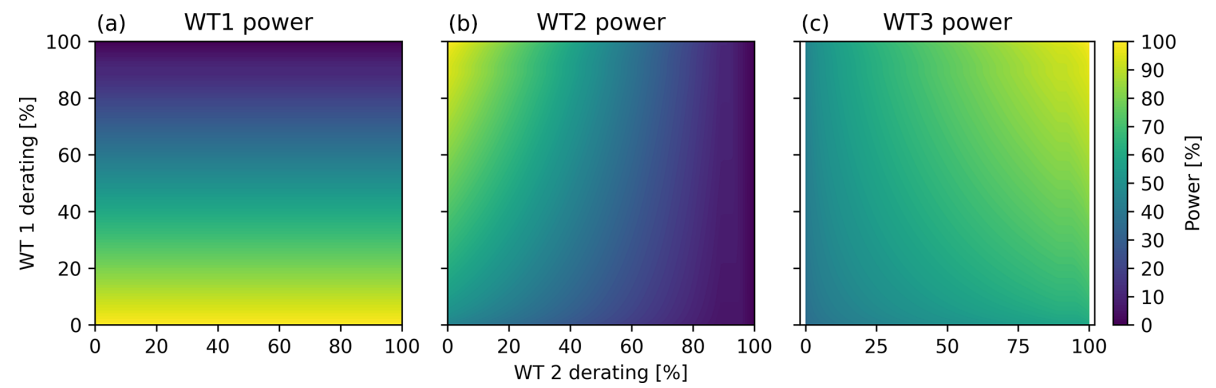

Figure 6. Power produced by the three WTs at $10 \mathrm{~m} \mathrm{~s}^{-1}$ as a function of WT1 and WT2 derating.

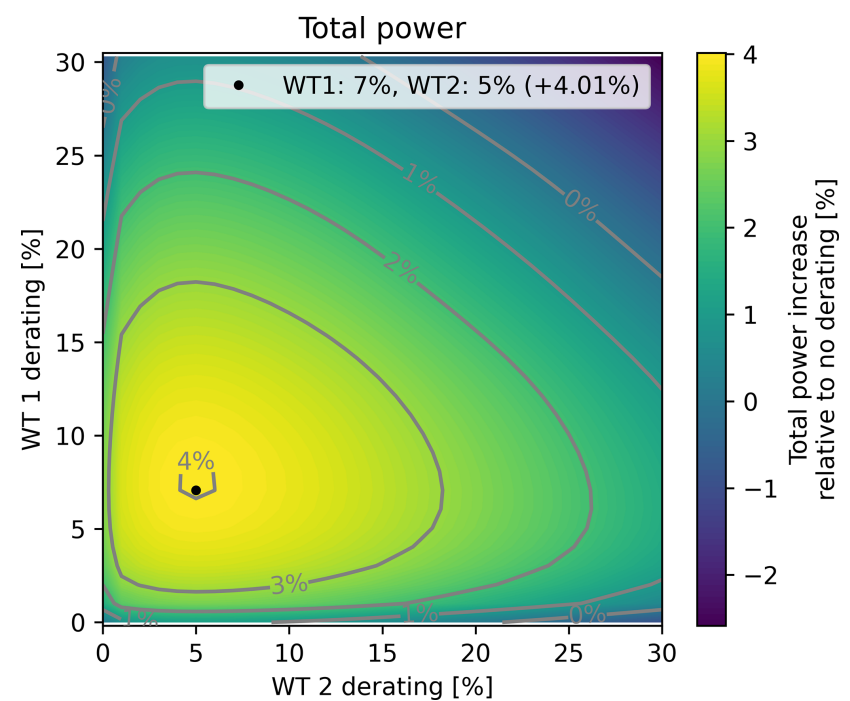

Figure 7. Total power of the three-WT row as a function of the derating of WT1 and WT2. The power can be increased by $4.01 \%$ when WT1 is derated by $7 \%$ and WT2 is derated by $5 \%$.

each with a rotor diameter of $93 \mathrm{~m}$. The WPP is known for its very small WT interspacings, down to $3.3 \mathrm{D}$ and associated pronounced wake effects. This makes this WPP especially suited for studies of WPP performance. The WPP layout is shown in Fig. 11.

The Lillgrund wind climate is outlined in Appendix A in terms of ambient mean wind speed pdfs (i.e. two-parameter

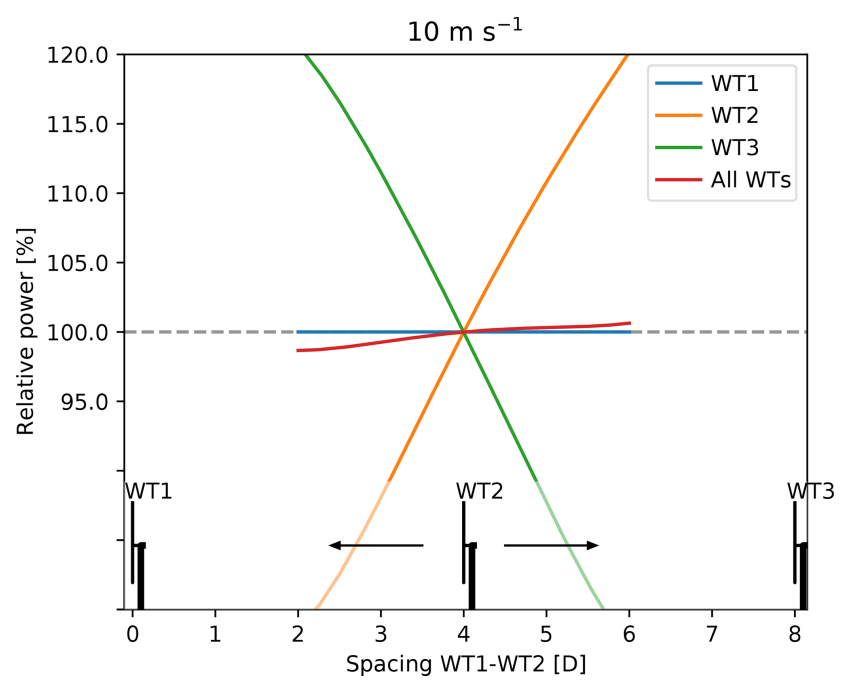

Figure 8. Relative power produced by the three individual WTs as well as the total relative power plotted as a function of the position of WT2.

Weibull), conditioned on the ambient mean wind direction as well as an ambient mean wind direction pdf. For the sake of illustration, the applied wind climate information is condensed in the wind rose shown in Fig. 12, which reveals predominant winds from the west and south.

First, we will focus on a subset of the Lillgrund WPP consisting of a row of eight WTs with along-row inflow conditions covering the entire relevant wind speed regime - i.e. the 


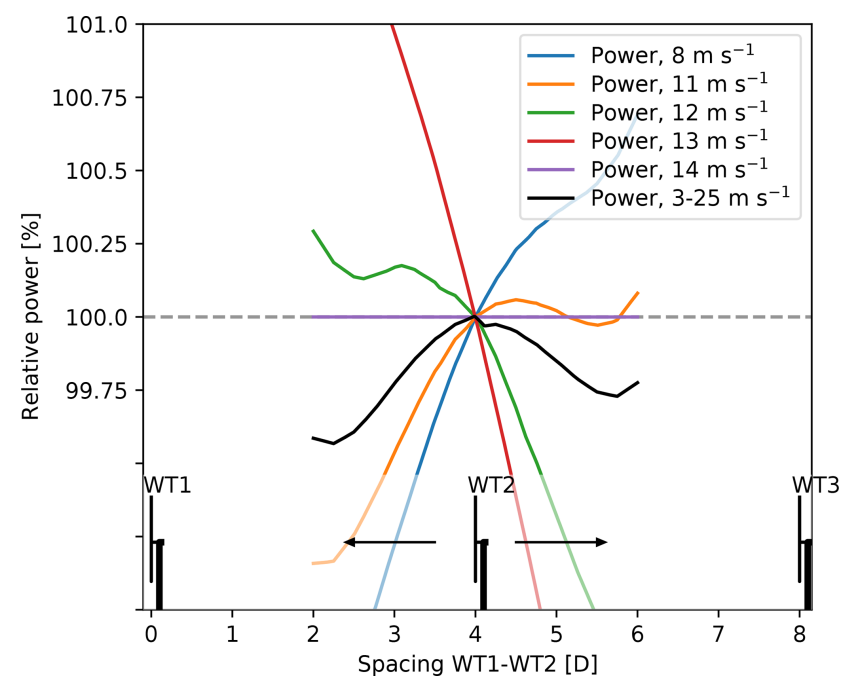

Figure 9. Relative total power production of the three WTs plotted as a function of the position of WT2 for different wind speeds. The power for $3-25 \mathrm{~m} \mathrm{~s}^{-1}$ is weighted by the Weibull distribution associated with wind from $270^{\circ}$.

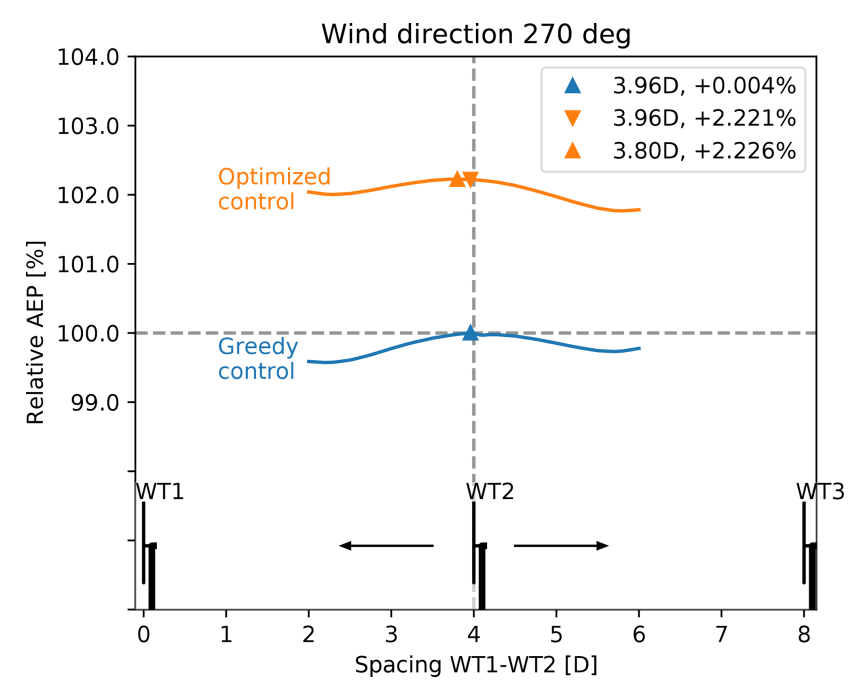

Figure 10. Relative AEP plotted as a function of the position of WT2 for both greedy and optimized control.

wind speed regime within which these WTs are in normal operation. Using this simplified case study, we will investigate the system coupling between WPP layout and WPP control optimization. Based on the results from this study, we will next perform a system optimization of the Lillgrund WPP and thereby quantify its potential in terms of increased AEP compared to the base case, which is the present layout (see Fig. 11) without coordinated WPP control - i.e. only the conventional greedy control of the individual WTs.

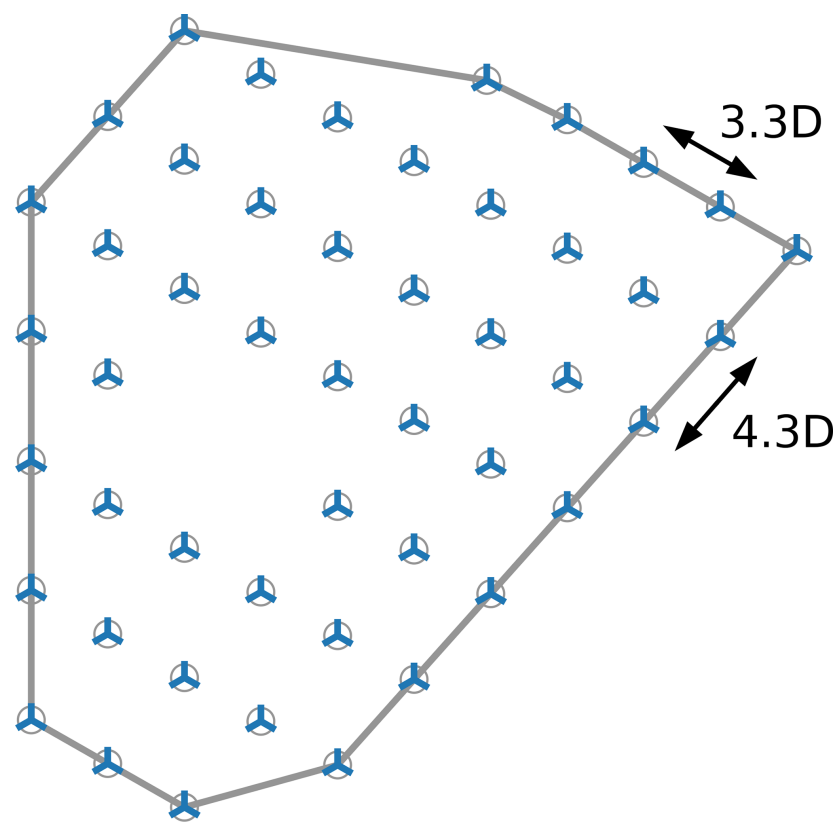

Figure 11. WT positions in the offshore Lillgrund WPP.

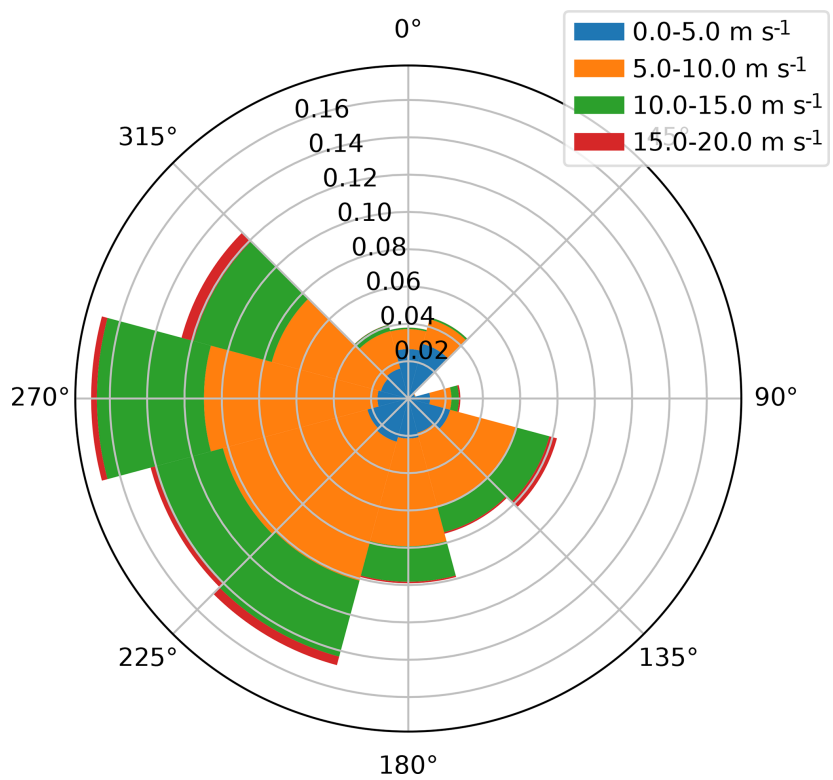

Figure 12. Wind rose characterizing the wind climate at the Lillgrund wind farm. Mean wind speed bins are shown in different colours, and their occurrence probabilities (conditioned on the respective inflow sectors) are proportional to their respective radial extents.

\subsection{Eight-WT row}

This case study basically consists of one of the three Lillgrund WWP rows with eight WTs, meaning that the WT interspacing in the base case is $3.3 \mathrm{D}$ (see Fig. 11) and that the WTs are Siemens SWT-2.3-93. The wind climate is fictitious, as only an along-row inflow direction is considered, 
which ensures the largest possible mutual WT wake interactions. Within this framework we have, without loss of generality, assumed Weibull-distributed mean wind speeds corresponding to the $270^{\circ}$ site condition (truncated, however, to the relevant wind speed regime $\left[3 \mathrm{~m} \mathrm{~s}^{-1}, 25 \mathrm{~m} \mathrm{~s}^{-1}\right]$ ) although the "true" inflow direction associated with this row is $300^{\circ}$.

With the purpose of investigating the strength of the system coupling, we have optimized the following: (1) the WPP layout, (2) the WPP control, (3) the integrated WPP layout and WPP control based on the two-step sequential approach (see Sect. 2.4), and (4) the integrated WPP layout and WPP control based on the two-step nested approach (see Sect. 2.4). Based on a pre-investigation of optimizers, where the random-search approach was compared to the SLSQP gradient-based optimization algorithm, the latter was found to be clearly superior and was consequently used in this study.

The results of the investigation are, together with the base case 0 , summarized in Table 2.

The base case, case 0 , represents the existing layout with the conventional greedy control of the individual WTs. Case 1 represents the base case layout with the WPP control optimized. The associated increase in AEP, relative to the base case, is significant and amounts to $8.0 \%$. The close spacing in the Lillgrund WPP case (3.3 D) is comparable with the WT interspacing (2.3-3.1 D) in the Goole Fields WPP investigated in van der Hoek et al. (2019), where an increase of $5.6 \%$ for a row of five WTs was predicted and an increase of $3.3 \%$ was realized in the accompanying full-scale study. As noted in the discussion of the results in this paper, the $k-\varepsilon$ turbulence closure of the CFD model, which was used for the tuning of the derating settings, supposedly makes the CFD predictions underestimate wake effects for closely spaced WTs, whereby the used pitch settings are likely to be suboptimal. Furthermore, only the first WT in the investigated row was derated, their two-step pitch-offset derating strategy was suboptimal, and finally the derating potential increases with the number of WTs. This, paired with the fact that full-scale studies will always suffer from imperfect inflow (due to, for example, wind direction variability within the 10 min recording sequences) and WT operational conditions (such as moderate yaw errors), makes us believe that the case 1 result is fairly consistent with the results presented in van der Hoek et al. (2019). In case 2 the WT applies the greedy control, and the WT row layout is optimized. The increase in AEP, relative to the base case, amounts to $1.4 \%$, which is considerably less than achieved in case 1. Compared to the three-WT case in Fig. 10, the AEP increase achieved by layout optimization in this case is much more pronounced because the number of design variables has increased from one to six. It is seen that the distances between the two most upstream and the three most downstream WTs are smaller than in the base case. This allows larger spacing and thereby production of the middle turbines, which, in this case, results in an increase in the AEP of the whole row. Obviously, this strategy is not possible with only three WTs. Case 3 represents one of two system optimization approaches. Here we assume that the system optimization is separable and consequently can be performed by first optimizing the layout and subsequently the WPP control. The combined effect is an increase in AEP amounting to $9.1 \%$, which is significant and exceeds what was obtained by only optimizing the WPP control (i.e. case 1). In the second and last system optimization strategy, case 4, the integrated two-step nested approach is taken. Although the approach is more complex and timeconsuming (around 540 times slower) than the case 3 strategy, the outcome is not significantly improved (see Table 2 ).

In conclusion, we have shown that the strength of the system coupling between WPP layout and WPP control optimization is only marginal for the considered eight-WT case study characterized by "heavy" mutual WT wake interactions.

\subsection{Full Lillgrund wind farm}

This case study comprises the entire Lillgrund WPP, and it ultimately aims to quantify the potential of an integrated system optimization of WPP layout and WPP control.

In analogy with Sect. 4.1, we will investigate a variety of WPP layout and WPP control optimization strategies. The control optimization schedule and the associated results appear in Table 3.

The investigated cases are analogous to the cases investigated for the eight-WT case in Sect. 4.1. As for case 1 we see a considerably drop in performance increase compared to the eight-WT situation, which is due to the persistently more severe mutual WT wake interactions in the fictitious eightWT situation compared to the full Lillgrund WPP, where WT wake interactions for some inflow directions are limited (see Fig. 14). With less wake interaction, less potential for WPP control follows intuitively. Case 2 represents an isolated WPP layout optimization retaining the greedy individual WT control performance. The associated increase in AEP performance amounts to $2.8 \%$ - or more than double that of the WPP control optimization, case 1 . The last case, case 3 , represents a system optimization approach. Based on the investigations performed in both Sects. 3.3 and 4.1, we assume that the system optimization is separable in the sense described in Sect. 4.1. The rationale justifying this assumption is that the system coupling between WPP layout and WPP control optimization was shown to be marginal in the eightWT case, in which the overall WT wake interaction, over all inflow directions, is significantly more pronounced than for the full Lillgrund case. Taking the sequential approach, the combined Lillgrund WPP optimization results in an AEP improvement of $4.0 \%$, which is significantly more than each of the individual layout and WPP control optimization approaches. Finally, it should be noted that, although possible, the two-step nested approach will require horrendous CPU 
Table 2. AEP results of various optimization approaches applied on the eight-WT row. The CPU times refer to the computation time on a standard laptop PC. The figures in the rightmost column show the position of the eight turbines. The derating settings of the WTs are indicated by the colour of the turbine symbol and quantified in percent by the number above the WT symbols.

\begin{tabular}{|c|c|c|c|c|c|}
\hline Case & Layout & Control & $\begin{array}{l}\text { AEP } \\
(\mathrm{GWh})\end{array}$ & $\begin{array}{l}\text { CPU } \\
\text { time } \\
\text { (s) }\end{array}$ & \\
\hline 0 & Initial & Greedy & 40.85 & 0.01 & 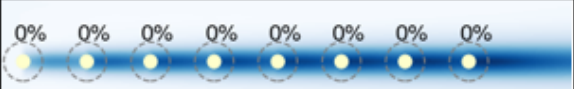 \\
\hline 1 & Initial & Optimized & $\begin{array}{l}44.10 \\
(+8.0 \%)\end{array}$ & 4.20 & 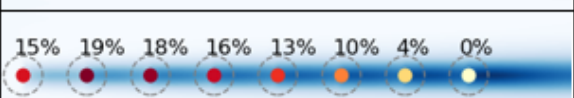 \\
\hline 2 & Optimized & Greedy & $\begin{array}{l}41.44 \\
(+1.4 \%)\end{array}$ & 2.84 & 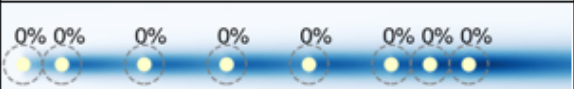 \\
\hline 3 & Optimized & $\begin{array}{l}\text { Optimized } \\
\text { (sequential) }\end{array}$ & $\begin{array}{l}44.558 \\
(+9.1 \%)\end{array}$ & 6.92 & 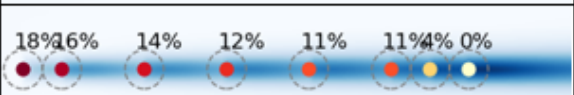 \\
\hline 4 & Optimized & Optimized (nested) & $\begin{array}{l}4.560 \\
(+9.1 \%)\end{array}$ & 3731 & $15 \% 6 \% \quad 14 \% \quad 13 \% \quad 11 \% \quad 11 \% \% 0 \%$ \\
\hline
\end{tabular}

Table 3. AEP results of various optimization approaches applied on the full Lillgrund WPP.

\begin{tabular}{lllr}
\hline Case & Layout & Control & AEP $(\mathrm{GWh})$ \\
\hline 0 & Initial & Greedy & 345.2 \\
1 & Initial & Optimized & $349.5(+1.3 \%)$ \\
2 & Optimized & Greedy & $354.9(+2.8 \%)$ \\
3 & Optimized & Optimized (sequential) & $358.7(+4.0 \%)$ \\
\hline
\end{tabular}

resources and even on a cluster take on the order of a few months to conduct.

The layout resulting from the Lillgrund WPP system optimization is shown in Fig. 13 together with the baseline layout.

From a pure production perspective, it makes sense to locate WTs densely at the boundary of the "admitted area" for the WPP, because it intuitively will reduce the WT wake interactions. Notable is also that the individual WT deratings for the shown example, except for one row, are considerably less than for the baseline case.

The results for all the investigated optimization strategies are summarized in Figs. 14 and 15. Figure 14 shows the increase in AEP conditioned on the inflow mean wind direction. As expected, the AEP gains vary with the wind direction with huge increases, up to $50 \%$, for the optimized layout for the wind directions that are parallel to the rows of the original layout, i.e. $120^{\circ} / 300^{\circ}, 42^{\circ} / 222^{\circ}$, and $0^{\circ} / 180^{\circ}$. These increases, however, are almost balanced out by the decrease in other directions resulting in the average increases of the $2.8 \%$ and $4 \%$ that are reported in Table 3 .
In Fig. 15, the AEP gains are shown as a function of the mean inflow wind speed. The largest increases are seen below $10-11 \mathrm{~m} \mathrm{~s}^{-1}$ where all WTs operate below rated power. At higher wind speeds, the WPP production wake losses decrease, as more and more WTs reach rated power, thus eventually completely eliminating any WPP control potential.

\section{Conclusions}

This paper describes a platform for integrated WPP layout and derating-based WPP control optimization. The objective function for the optimization is the AEP of the WPP without considering financial costs of the internal WPP grid. This means that the positions of the individual WPP WTs are only constrained by a minimum allowable distance to the nearest neighbouring WT, in this case $2 \mathrm{D}$, and the convex boundary around the initial WPP layout.

As WPP loading is excluded, stationary modelling of the complex WPP flow field suffices, which is a considerable simplification. Contrary to other known WPP optimization platforms, the present approach is based on a consistent and very fast CFD solver, whereby the inherent uncertainties associated with simple empirical algebraic wake models, including their often debatable wake summation procedure, are avoided. This strategy is consistent with a recent review of WPP optimization approaches (Kheirabadi and Nagamune, 2019), where one of the conclusions is that "added layers of realism in terms of simulated wind conditions tend to deteriorate the performance of wind farm controllers", thus stressing the importance of carefully and realistically simulated WPP flow fields. 
Initial layout, WS: $10 \mathrm{~m} \mathrm{~s}^{-1}$, WD: $223^{\circ}$ Power 43.43MW $(+6.61 \%)$

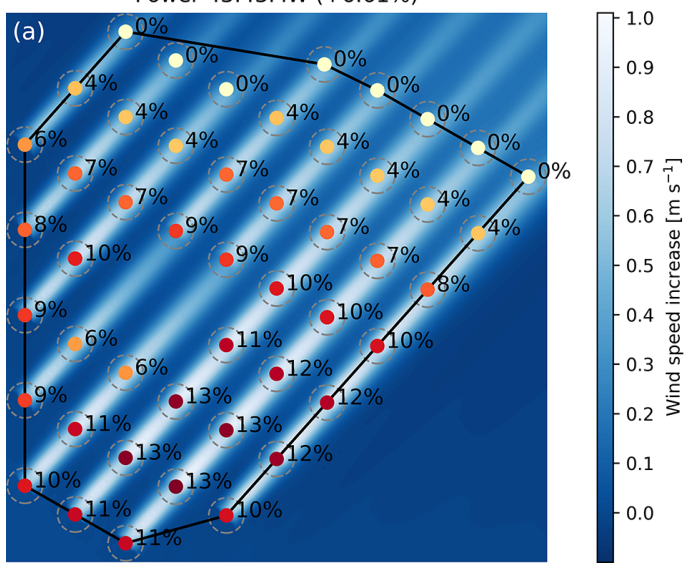

Optimized layout, WS: $10 \mathrm{~m} \mathrm{~s}^{-1}, \mathrm{WD}: 223^{\circ}$

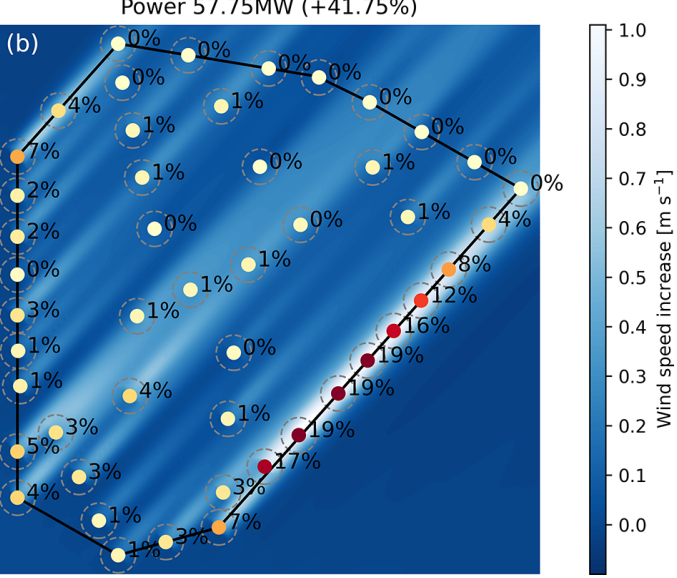

Figure 13. The baseline Lillgrund WPP layout (a) and optimized Lillgrund WPP layout (b). The two panels show the flow case associated with $10 \mathrm{~m} \mathrm{~s}^{-1}$ inflow from direction $223^{\circ}$. The derating settings of the individual WTs are indicated by the colour of the WT symbols and quantified in percent by the number above the WT symbols. The background colours illustrate the increase in wind speed from the individual greedy to the collaborative optimized control situation.

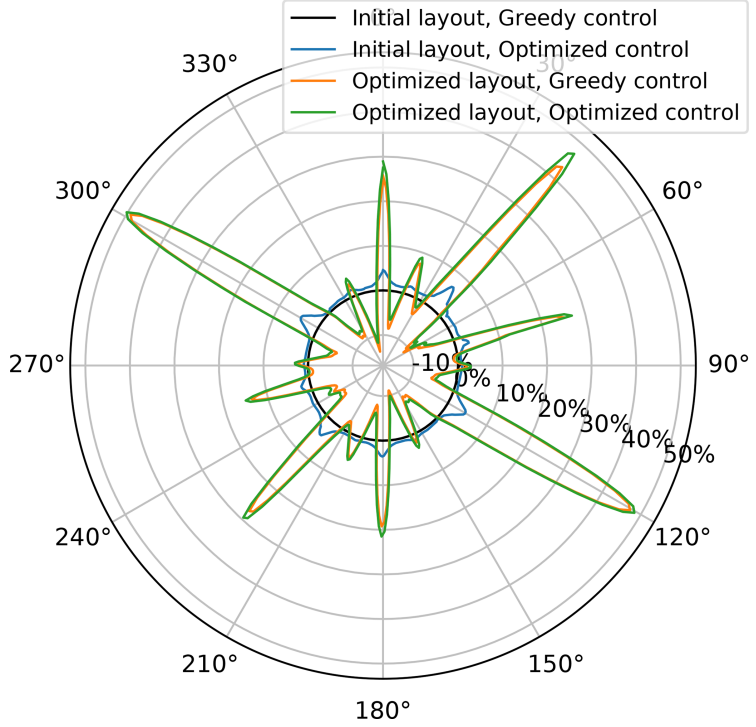

Figure 14. Increase in AEP due to layout and/or control optimization plotted as a function of inflow wind direction.

The platform has initially successfully been subjected to a simplistic sanity check. Subsequently, the platform has been used to analyse the potential of an integrated WPP layout and WPP control optimization of the offshore WPP Lillgrund, which consists of 48 closely spaced WTs. First, an analysis of the system coupling between WPP layout optimization and WPP control optimization is performed as based on a subset of this WPP exposed to inflow conditions clearly exaggerating the overall complex inter-WT aerodynamic interactions within a traditional WPP, because all the WTs are in a state of maximum wake interaction. The study demonstrates

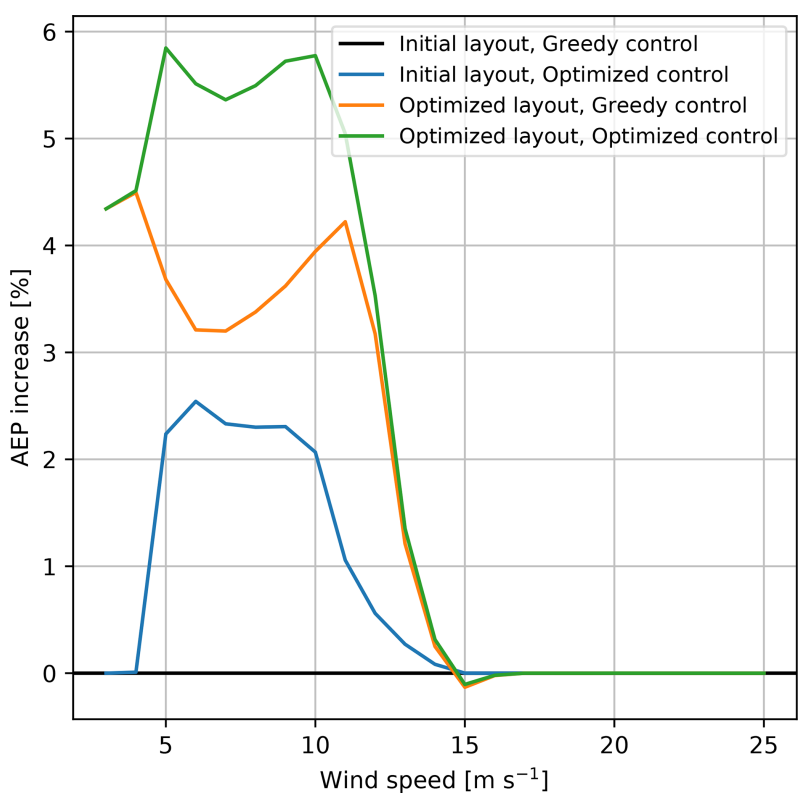

Figure 15. Increase in AEP due to layout and/or control optimization plotted as a function of wind speed.

an inferior system coupling only, thus justifying separation of the present optimal system design. Based on this learning, a full system optimization of the Lillgrund WPP is performed, resulting in a gain amounting to $4.0 \%$ in AEP relative to the baseline case, which is the present Lillgrund layout without WPP control.

In a future perspective, the platform will be extended to also include active wake control in terms of WT yaw-dictated wake deflection. This requires a generalization of the applied linearized CFD flow solver Fuga - a work that is in progress. 
Appendix A

Table A1. Sector probability and Weibull shape and scale parameters for the Lillgrund site. Data obtained from the study of Göçmen and Giebel (2016).

\begin{tabular}{lrrr}
\hline $\begin{array}{l}\text { Wind sector } \\
\left(\text { centred; }{ }^{\circ}\right)\end{array}$ & $\begin{array}{r}\text { Frequency } \\
(\%)\end{array}$ & $\begin{array}{r}\text { Weibull scale } \\
(A)\end{array}$ & $\begin{array}{r}\text { Weibull shape } \\
(k)\end{array}$ \\
\hline 0 & 3.8 & 4.5 & 1.69 \\
30 & 4.5 & 4.7 & 1.78 \\
60 & 0.4 & 3.0 & 1.82 \\
90 & 2.8 & 7.2 & 1.7 \\
120 & 8.3 & 8.8 & 1.97 \\
150 & 7.5 & 8.2 & 2.49 \\
180 & 9.9 & 8.4 & 2.72 \\
210 & 14.8 & 9.5 & 2.7 \\
240 & 14.3 & 9.2 & 2.88 \\
270 & 17.0 & 9.9 & 3.34 \\
300 & 12.6 & 10.3 & 2.84 \\
330 & 4.1 & 6.7 & 2.23 \\
\hline
\end{tabular}


Data availability. Simulation data are not available due to the confidentiality of the Siemens WT model.

Author contributions. GCL designed the numerical study. MMP implemented and ran the optimizations. The paper was written and reviewed in cooperation.

Competing interests. The authors declare that they have no conflict of interest.

Special issue statement. This article is part of the special issue "Wind Energy Science Conference 2019". It is a result of the Wind Energy Science Conference 2019, Cork, Ireland, 17-20 June 2019.

Acknowledgements. Siemens Gamesa Renewable Energy is acknowledged for making the aerodynamic data of the Siemens SWT2.3-93 WT available for the Lillgrund study.

Financial support. This research has been supported by the European Commission, H2020 Research Infrastructures (TotalControl (grant no. 727680)).

Review statement. This paper was edited by Julie Lundquist and reviewed by two anonymous referees.

\section{References}

Andersen, S. J.: A Comparative Study of the Wake Dynamics during Yaw and Curtailment, Zenodo, https://doi.org/10.5281/zenodo.3357798, 2019.

Deshmukh, A. P. and Allison, J. T.: Unrestricted wind Farm Layout Design with Optimal Control Considerations, in: Proceedings of the ASME 2017 International Design Engineering Technical Conferences and Computers and Information in Engineering Conference, Cleveland, Ohio, USA, August 6-9, 2017.

Fathy, H. K., Reyer, J. A., Papalambros, P. Y., and Ulsoy, A. G.: On the coupling between the plant and controller optimization problems, Proceedings of the 2001 American Control Conference. (Cat. No.01CH37148), Arlington, VA, USA, 25-27 June 2001, IEEE, 3, 1864-1869, https://doi.org/10.1109/ACC.2001.946008, 2001.

Feng, J. and Shen, W. Z.: Solving the wind farm layout optimization problem using random search algorithm, Renew. Energ., 78, 182-192, https://doi.org/10.1016/j.renene.2015.01.005, 2015.

Fleming, P. A., Ning, A., Gebraad, P. M. O., and Dykes, K.: Wind plant system engineering through optimization of layout and yaw control, Wind Energy, 19, 329-344, https://doi.org/10.1002/we.1836, 2016.

Gebraad, P. M. O., Fleming, P. A., and van Wingerden, J. W.: Comparison of actuation methods for wake control in wind plants, American Control Confer- ence (ACC), Chicago, IL, 1-3 July 2015, 1695-1701, https://doi.org/10.1109/ACC.2015.7170977, 2015.

Gebraad, P. M. O., Thomas, J. J., Ning, A., Fleming, P. A., and Dykes, K.: Maximization of the annual energy production of wind power plants by optimization of layout and yaw-based wake control, Wind Energy, 20, 97-107, https://doi.org/10.1002/we.1993, 2017.

Göçmen, T. and Giebel, G.: Estimation of turbulence intensity using rotor effective wind speed in Lillgrund and Horns Rev-I offshore wind farms, Renew. Energ., 99, 524-532, https://doi.org/10.1016/J.RENENE.2016.07.038, 2016.

Gray, J. S., Hwang, J. T., Martins, J. R. R. A., Moore, K. T., and Naylor B. A.: OpenMDAO: An Open-Source Framework for Multidisciplinary Design, Analysis, and Optimization, Struct. Multidiscip. O., 59, 1075-1104, https://doi.org/10.1007/s00158019-02211-z 2019.

Hansen, M. H., Henriksen, L. C., Tibaldi, C., Bergami, L., Verelst, D., and Pirrung, G.: HAWCStab2 User Manual, Department of Wind Energy Report 2017, DTU Wind Energy, 2017.

Jensen, N. O.: A note on wind generator interaction, Ris $\varnothing-M-2411$, Risø National Laboratory, Roskilde, 1984.

Kheirabadi, A. C. and Nagamune, R.: A quantitative review of wind farm control with the objective of wind farm, J. Wind Eng. Ind. Aerod., 192, 45-73, 2019.

Larsen, G. C. and Réthoré, P.-E.: TOPFARM - A Tool for Wind Farm Optimization, Energy Proced., 35, 317-324, 2013.

Machefaux, E., Larsen, G. C., Troldborg, N., Gaunaa M., and Rettenmeier, A.: Empirical Modelling of Single Wake Advection and Expansion using Full Scale Pulsed Lidar based Measurements, Wind Energy, 18, 2085-2103, 2015.

Madsen, H. A., Mikkelsen, R., Sørensen, N. N., Hansen, M. L. O., $\emptyset$ ye, S., and Johansen, J.: Influence of wind shear on rotor aerodynamics, power and loads, in: Research in Aeroelasticity EFP2006, edited by: Bak, C., Ris $\varnothing$ National Laboratory, Denmark, Forskningscenter Risoe, Ris $\varnothing-R$, no. 1611(EN), 2007.

Mahulja, S., Larsen, G. C., and Elham, A.: Engineering an optimal wind farm using surrogate models, Wind Energy, 21, 1296-1308, 2018.

Ott, S., Berg, J., and Nielsen, M.: Linearised CFD Models for Wakes, Danmarks Tekniske Universitet, Risø Nationallaboratoriet for Bæredygtig Energi, Denmark, Forskningscenter Risoe, Risoe-R, no. 1772(EN), 2011.

Peña, A., Schaldemose Hansen, K., Ott, S., and van der Laan, M. P.: On wake modeling, wind-farm gradients, and AEP predictions at the Anholt wind farm, Wind Energ. Sci., 3, 191-202, https://doi.org/10.5194/wes-3-191-2018, 2018.

Pedersen, M. M., Friis-Møller, M., and Réthoré, P.-E.: DTUWindEnergy/TopFarm2: Topfarm2 v2.1.0, Zenodo, https://doi.org/10.5281/zenodo.3247032, 2019a.

Pedersen, M. M., van der Laan, P., Friis-Møller, M., Rinker, J., and Réthoré, P.-E.: DTUWindEnergy/PyWake: PyWake, Zenodo, https://doi.org/10.5281/zenodo.2562662, 2019 b.

Réthoré, P.-E., Fuglsang, P., Larsen, G. C., Buhl, T., Larsen, T. J., and Madsen, H. A.: TOPFARM: Multi-fidelity optimization of wind farms, Wind Energy, 17, 1797-1816, 2013.

van der Hoek, D., Kanev, S., Allin, J., Bieniek, D., and Mittelmeier, N.: Effects of axial induction control on wind farm energy production - a field test, Renew. Energ., 140, 994-1003, https://doi.org/10.1016/j.renene.2019.03.117, 2019. 
van der Laan, M. P., Andersen, S. J., Ramos García, N., Angelou, N., Pirrung, G. R., Ott, S., Sjöholm, M., Sørensen, K. H., Vianna Neto, J. X., Kelly, M., Mikkelsen, T. K., and Larsen, G. C.: Power curve and wake analyses of the Vestas multi-rotor demonstrator, Wind Energ. Sci., 4, 251-271, https://doi.org/10.5194/wes4-251-2019, 2019.
Vitulli, J. A., Larsen, G. C., Pedersen, M. M., Ott, S., and Friis-Møller, M.: Optimal open loop wind farm control, J. Phys. Conf. Ser., 1256, 012027, https://doi.org/10.1088/17426596/1256/1/012027, 2019. 